OPEN ACCESS

Edited by:

Deqiang Qiu,

Emory University, United States

Reviewed by:

Pilar Maria Ferraro,

IRCCS Ospedale Policlinico San

Martino, Italy

Alessia Sarica,

University of Magna Graecia, Italy

*Correspondence:

Yi Lei

13602658583@163.com

Fan Lin

foxetfoxet@gmail.com

tThese authors have contributed equally to this work and share first authorship

Specialty section:

This article was submitted to Applied Neuroimaging, a section of the journal Frontiers in Neurology

Received: 02 December 2020 Accepted: 05 February 2021

Published: 25 February 2021

Citation:

Zhang $H$, He W-J, Liang L-H, Zhang $H$-W, Zhang $X$-J, Zeng $L$, Luo S-P, Lin F and Lei Y (2021)

Diffusion Spectrum Imaging of Corticospinal Tracts in Idiopathic Normal Pressure Hydrocephalus. Front. Neurol. 12:636518. doi: 10.3389/fneur.2021.636518

\section{Diffusion Spectrum Imaging of Corticospinal Tracts in Idiopathic Normal Pressure Hydrocephalus}

\author{
Hong Zhang ${ }^{1,2 t}$, Wen-Jie He ${ }^{1 \dagger}$, Li-Hong Liang ${ }^{1}$, Han-Wen Zhang ${ }^{1}$, Xie-Jun Zhang ${ }^{3}$, \\ Liang Zeng ${ }^{1}$, Si-Ping Luo ${ }^{1,2}$, Fan Lin ${ }^{1 *}$ and Yi Lei ${ }^{1 *}$ \\ 1 Department of Radiology, Health Science Center, Shenzhen Second People's Hospital, The First Affiliated Hospital of \\ Shenzhen University, Shenzhen, China, ${ }^{2}$ Shantou University Medical College, Shantou, China, ${ }^{3}$ Department of Neurosurgery, \\ Health Science Center, Shenzhen Second People's Hospital, The First Affiliated Hospital of Shenzhen University, Shenzhen, \\ China
}

Purpose: The purpose of this study was to measure the diffusion spectrum imaging (DSI) parameters of corticospinal tracts (CSTs) and evaluate diffusional changes in CSTs in patients with idiopathic normal pressure hydrocephalus (iNPH) by DSI.

Methods: Twenty-three iNPH patients and twenty-one healthy controls (HCs) were involved in this study. Brain DSI data for all participants were collected through the same MR scanning procedure. The diffusion parameters measured and analyzed included quantitative anisotropy (QA), the isotropic diffusion component (ISO), general fractional anisotropy (GFA), fractional anisotropy (FA), mean diffusivity (MD), axial diffusivity (AD), and radial diffusivity $(\mathrm{RD})$ of corticospinal tracts.

Results: The QA and ISO values of corticospinal tracts in iNPH patients were significantly lower than those in HCs $\left(P_{L Q A}=0.008, P_{R Q A}=0.016, P_{\text {LISO }}=0.024, P_{\text {RISO }}=0.016\right)$. The mean $M D, A D$, and $R D$ values in $\mathrm{NPH}$ patients were significantly higher than those in $\mathrm{HCs}\left(\mathrm{P}_{\mathrm{MD}}=0.032, \mathrm{P}_{\mathrm{AD}}=0.032, \mathrm{P}_{\mathrm{RD}}=0.048\right.$,). No significant differences in GFA and FA values were noted between iNPH patients and HCs.

Conclusion: Decreased QA and ISO values of corticospinal tracts were found in iNPH patients. Quantitative CST evaluation using DSI may lead to information that can improve the present understanding of the disease mechanism.

Keywords: idiopathic normal pressure hydrocephalus, diffusion spectrum imaging, corticospinal tract, quantitative anisotropy, diffusion magnetic resonance image

\section{INTRODUCTION}

Idiopathic normal pressure hydrocephalus (iNPH) is a clinical syndrome that occurs in elderly people (1). Although the etiology remains unclear, it is a syndrome characterized by progressive gait disorder, cognitive impairment, and urinary incontinence $(2,3)$. Patients present with ventricular enlargement detected by radiological assessment with a normal range of cerebrospinal fluid (CSF) pressure $(4,5)$. Surgical shunt surgery is considered an effective treatment for iNPH $(6)$.

Gait disorders are the most common and most characteristic symptom of iNPH, the mechanism of which is widely explained as enlarged ventricles mechanically compressing the paraventricular nerve fibers, especially corticospinal tracts (CSTs) (7). Previous studies used diffusion tensor 
imaging (DTI) to investigate subcortical involvement in the brains of iNPH patients and attempted to demonstrate white matter integrity changes (8). However, DTI cannot be used to evaluate the crossing and twining fiber bundles because it depends on a Gaussian parameterization of diffusion (9). Recently, diffusion spectrum imaging (DSI), a new kind of diffusion magnetic resonance imaging (dMRI) technique, has been used to noninvasively detect the complex white matter tract architecture and structural changes of fiber tracts in the human brain (10). DSI has been used to study some mental disorders such as autism and schizophrenia and neurodegenerative diseases such as Alzheimer's disease and multiple sclerosis (11-14). DSI reconstructs fiber tracts with a much higher resolution than conventional DTI and has been proven to accurately display crossing, winding, interrupted and small fibers $(15,16)$. The general fractional anisotropy (GFA) value is the main quantitative parameter of DSI, representing the directional consistency of water molecule diffusion (17). Generalized q-sampling imaging (GQI) (18) is one of the common reconstruction models for DSI data that can be used to calculate the indexes of quantitative anisotropy (QA) and the isotropic diffusion component (ISO) in addition to GFA.

However, the application of DSI in iNPH patients has not been reported at present. Thus, the purpose of this study was to evaluate diffusional changes in the corticospinal tract (CST) in patients with idiopathic normal pressure hydrocephalus by DSI.

\section{MATERIALS AND METHODS \\ Demographics and Clinical Data}

This study included 23 defined iNPH patients according to the guidelines for iNPH who were treated in the neurosurgery department of our hospital between March 2019 and May 2020 (1). Figure 1 shows the flow chart of the selection process for the iNPH and HC groups. Forty-two patients were included based on the following criteria: older than 60 years of age, presenting with one or more other typical triad symptoms (gait disturbance, cognitive disorder, and urinary incontinence), and the presence of a short-term radiology imaging finding indicating ventricular enlargement (19). Thirteen of these patients were excluded due to obstructive hydrocephalus $(n=6)$, no CSF shunting $(n=4)$, and abnormal CSF pressure $(n=3)$. Six patients with a clear history of neurologic tumors $(n=3)$, cerebral hemorrhage $(n=$ $3)$, intracranial inflection $(n=2)$, and craniocerebral trauma ( $n$ $=1$ ) that causes structural and functional changes in the brain were excluded. All recruited patients underwent the same MRI protocol and clinical assessments before CSF shunting.

The group of healthy controls (HCs) was recruited by placing advertisements in several health management centers and asking elderly individuals (over 60 years old) without a definite history of serious diseases such as ischemic or hemorrhagic stroke, trauma,

Abbreviations: $\mathrm{P}_{\mathrm{LQA}}, p$ value for $\mathrm{QA}$ metrics of the left CST; $\mathrm{P}_{\mathrm{RQA}}, p$-value for $\mathrm{QA}$ metrics of the right CST; $\mathrm{P}_{\text {LISO }} p$-value for ISO metrics of the left CST; $\mathrm{P}_{\mathrm{RISO}}, p$ value for ISO metrics of the right CST; $\mathrm{P}_{\mathrm{MD}}, p$-value for $\mathrm{MD}$ metrics; $\mathrm{P}_{\mathrm{AD}}, p$-value for $\mathrm{AD}$ metrics; $\mathrm{P}_{\mathrm{RD}}$, $p$-value for $\mathrm{RD}$ metrics.

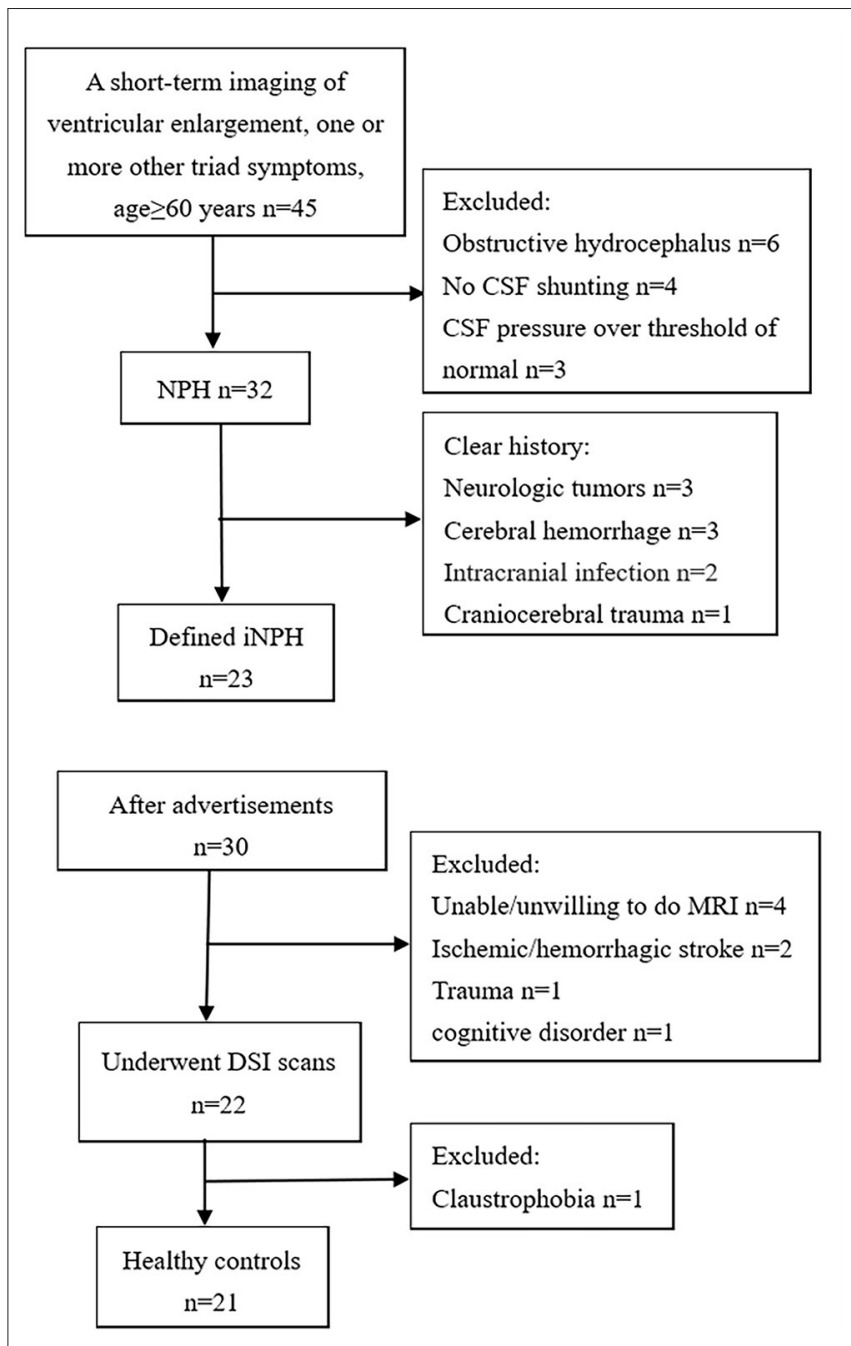

FIGURE 1 | Flow chart of selection for the iNPH and HC groups in this study.

or cognitive disorder to participate in this study. After historytaking and reading their imaging, 21 age- and sex-matched healthy volunteers were recruited as the HC group. All of the participants received the same brain MRI scans as the iNPH group. Age was included as the covariate in the statistical analysis because it was sensitive to the diffusion MRI indexes. Informed consent documents were signed prior to the examination by both groups of subjects. The protocol was approved by the Hospital Bioethics Committee.

\section{MR Imaging Acquisition}

All subjects underwent MRI examination on a 3-Tesla MR scanner (Prisma; Siemens; Erlangen, Germany) with 20-channel phase-array head coils. The iNPH group was scanned before lumbar drainage or ventricular shunt surgery. The participants' heads were to remain stationary during the scanning as much as possible or with the help of a fixator if necessary. 
TABLE 1 | Demographic data and clinical characteristic of the iNPH patients and HCs.

\begin{tabular}{|c|c|c|c|}
\hline Characteristics & iNPH patients $(n=23)$ & $\mathrm{HC}(n=21)$ & P-value \\
\hline Gender, male & $13(56.5)$ & $11(52.4)$ & 0.723 \\
\hline Age (year) & $70.2 \pm 9.1$ & $69.7 \pm 8.8$ & 0.146 \\
\hline \multicolumn{4}{|l|}{ Symptoms present } \\
\hline Triad of symptoms & $13(56.5)$ & $\mathrm{N} / \mathrm{A}$ & \\
\hline Gait + cognitive impairment & $8(34.8)$ & $\mathrm{N} / \mathrm{A}$ & \\
\hline Gait + urinary symptom & 0 & $\mathrm{~N} / \mathrm{A}$ & \\
\hline Cognitive impairment + urinary symptom & 0 & $\mathrm{~N} / \mathrm{A}$ & \\
\hline Gait only & $2(8.7)$ & $\mathrm{N} / \mathrm{A}$ & \\
\hline Cognitive impairment only & 0 & $\mathrm{~N} / \mathrm{A}$ & \\
\hline Urinary symptom only & 0 & $\mathrm{~N} / \mathrm{A}$ & \\
\hline
\end{tabular}

Data are presented as number (\%) of patients.

N/A, Not Applicable.

Data were collected using MRI sequences including T1weighted magnetization-prepared rapid acquisition gradientecho (MPRAGE) for better anatomic reference and diffusion spectrum imaging (DSI) using pulsed gradient twice-refocused spin-echo echo-planar imaging (EPI) sequences. T1-weighted sagittal images were obtained with 3D fast-field echo imaging. The following acquisition parameters were employed: repetition time, 2,300 ms; echo time, $3.55 \mathrm{~ms}$; flip angle, $8^{\circ}$; slice thickness, $0.9 \mathrm{~mm}$; number of sections, 192; field of view (FOV), $240 \times$ $240 \mathrm{~mm}$; matrix, $256 \times 256$; and voxel size, $0.9 \mathrm{~mm} \times 0.9 \mathrm{~mm}$. The acquisition parameters for DSI were as follows: repetition time, $6,300 \mathrm{~ms}$; echo time, $71 \mathrm{~ms}$; slice thickness, $2.2 \mathrm{~mm}$; number of sections in the transverse plane, 60; field of view (FOV), 220 $\times 100 \mathrm{~mm}$; matrix, $220 \times 100$; voxel size, $2.2 \mathrm{~mm} \times 2.2 \mathrm{~mm}$; and total diffusion sampling, 128, with a maximum diffusion sensitivity ( $b$-values max) of $3,000 \mathrm{~s} / \mathrm{mm}^{2}$.

\section{Tract Analysis}

To analyze the microstructural integrity of the white matter fiber tracts, we used the DSI studio software (http://dsi-studio. labsolver.org/) to reconstruct the corticospinal tract (CST) of all participants. The diffusion MR images were imported in the DSI studio software, setting up the brain mask and using the generalized q-sampling imaging (GQI) reconstruction model (18) with a diffusion sampling length ratio of 1.25. Restricted diffusion was quantified using restricted diffusion imaging (20). CSTs were generated by manually placing seeds in the precentral motor cortex and placing targets in the cerebral peduncles (21). The volume sizes of the seeds were $2.8 \times 10^{3} \mathrm{~mm}^{3}$ (left) and $3.0 \times 10^{3} \mathrm{~mm}^{3}$ (right). The volume sizes of the targets were $1.3 \times 10^{3} \mathrm{~mm}^{3}$ (left) and $1.4 \times 10^{3} \mathrm{~mm}^{3}$ (right). These seeds and targets were adjusted to the precentral motor cortex and cerebral peduncles of each person to kept constant in all participants. A deterministic fiber tracking algorithm was used to reconstruct the CSTs (22). The angular threshold was 75 degrees. The step size was $1 \mathrm{~mm}$. Tracks with lengths shorter than 20 or longer than $300 \mathrm{~mm}$ were discarded. A total of 5,000 tracts were calculated. Finally, the mean general fractional anisotropy (GFA), quantitative anisotropy (QA), normalized quantitative anisotropy (NQA), and isotropic diffusion component (ISO) values were calculated, as well as the elementary DTI parameters including fractional anisotropy (FA), mean diffusivity (MD), axial diffusivity (AD), and radial diffusivity (RD). The parameters of the right and left hemispheres were averaged for analysis if they had no significant difference and were analyzed separately if they had significant differences (21). Two radiologists with more than 10 years of experience in neuroradiology traced the seeds and targets separately and then evaluated the intergroup correlation coefficient of DSI parameters between the two tracers.

\section{Statistical Analysis}

All statistical analyses were performed using SPSS version 25.0 software. One-way ANCOVA was used to compare the differences in age and sex between the iNPH patients and HCs. The Wilcoxon matched-pairs signed-ranks test was performed to test the differences in DSI-relevant values in the two hemispheres. The Mann-Whitney $U$-test was used to compare the differences in DSI values between the iNPH and healthy control groups. Bonferroni correction was used to correct the multiple comparisons of different groups. The adjusted statistical significance level was accepted at 0.05 (two-tailed).

\section{RESULTS}

In total, 23 defined iNPH patients (age 70.2 \pm 9.1 years; range 6081 years; 13 men and 10 women) and 21 healthy volunteers (age $69.7 \pm 8.8$ years; range $60-80$ years; 11 men and 10 women) were included in this study. Sex and age were not significantly different between the two groups of subjects. The objective symptoms on the iNPH Grading Scales were gait disturbance in $100 \%$ of the patients, cognitive impairment in $91.3 \%$ of patients, urinary symptoms in $56.5 \%$ of patients, and the triad of symptoms in $56.5 \%$ of patients (Table 1). All of the patients presented with varying degrees of gait disturbance.

Figure 2 shows the parametric maps of the QA, ISO, MD, $\mathrm{AD}$, and color-FA values of the $\mathrm{iNPH}$ and $\mathrm{HC}$ samples. After tracking and reconstructing the corticospinal tracts of both hemispheres in each subject, the DSI parameters of the 


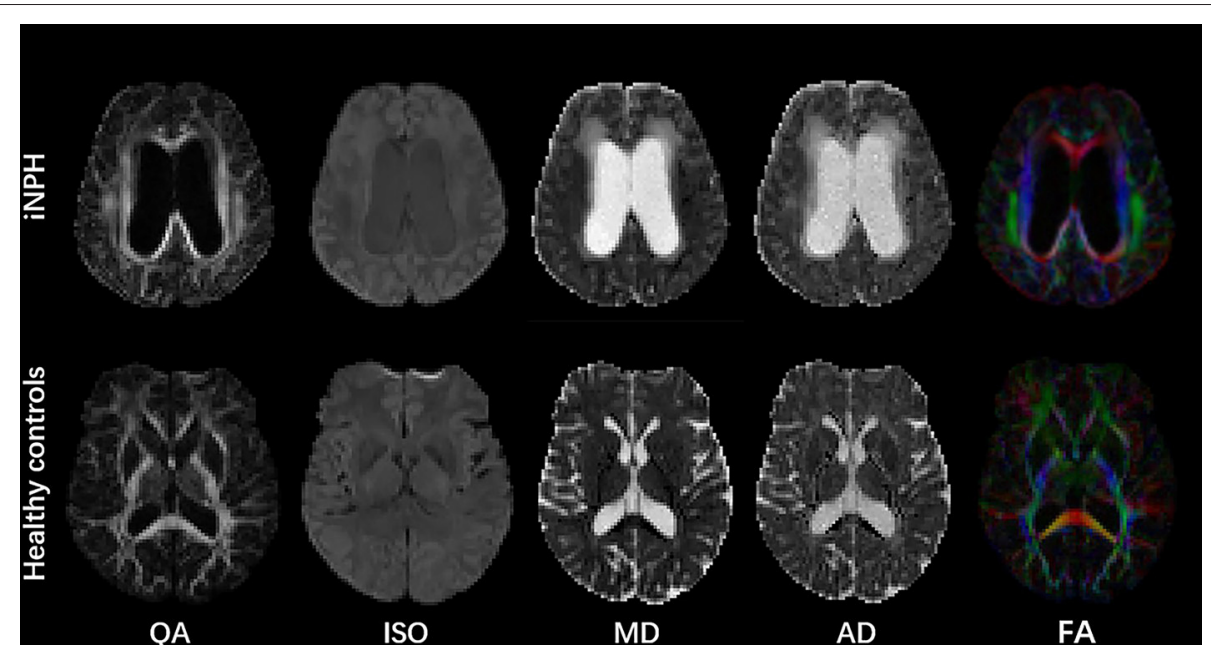

FIGURE 2 | Parametric maps of QA, ISO, MD, AD, and color FA values of the $\mathrm{iNPH}$ patient and HC samples.

whole tracts were calculated separately. Two neuroradiologists measured and calculated the parameters separately, and the intergroup correlation coefficients (ICCs) of these measurements were ICC $\mathrm{QA}=0.92, \mathrm{ICC}_{\mathrm{ISO}}=0.96, \mathrm{ICC}_{\mathrm{MD}}=0.93, \mathrm{ICC}_{\mathrm{AD}}$ $=0.92, \mathrm{ICC}_{\mathrm{FA}}=0.89, \mathrm{ICC}_{\mathrm{RD}}=0.90, \mathrm{ICC}_{\mathrm{GFA}}=0.92$, and $\mathrm{ICC}_{\mathrm{NQA}}=0.90$. The parameters were averaged and analyzed. Figure 3 shows the sample of corticospinal tracts reconstructed by deterministic tracking for patients with iNPH and healthy controls. The mean QA values and mean ISO values were not significantly different between the right hemisphere and left hemisphere $\left(\mathrm{P}_{\mathrm{QA}}=0.147, \mathrm{P}_{\mathrm{ISO}}=0.159\right)$ in iNPH patients, while the HCs had significant differences between the two hemispheres $(P=0.006,0.002$, respectively). Other mean values of FA, MD, $\mathrm{AD}, \mathrm{RD}$, and GFA did not significantly differ between the two sides of hemispheres.

Table 2 shows the different values measured from corticospinal tracts and the significant difference between iNPH patients and healthy controls. Compared with the healthy volunteers, the QA and ISO values of the two sides of the corticospinal tracts were both significantly reduced in iNPH patients $\left(\mathrm{P}_{\mathrm{LQA}}=0.008, \mathrm{P}_{\mathrm{RQA}}=0.016, \mathrm{P}_{\mathrm{LISO}}=0.024\right.$, and $\mathrm{P}_{\mathrm{RISO}}$ $=0.016$ ). The mean $\mathrm{MD}, \mathrm{AD}$, and $\mathrm{RD}$ values were all significantly different between iNPH patients and healthy controls. Mean MD, $\mathrm{AD}$, and $\mathrm{RD}$ values in $\mathrm{iNPH}$ patients were increased compared with those of HCs. The differences in the QA, ISO, MD, and AD values of the two groups are presented in Figure 4. Regarding the mean FA and GFA values, there was no significant difference between iNPH patients and healthy people.

\section{DISCUSSION}

In this study, we explored the corticospinal tract DSI parameters in patients with iNPH. We found that among the DSI parameters, the QA values, and ISO values of the CSTs for both the left and right sides were significantly different from those of healthy controls. On the other hand, regarding the traditional DTI

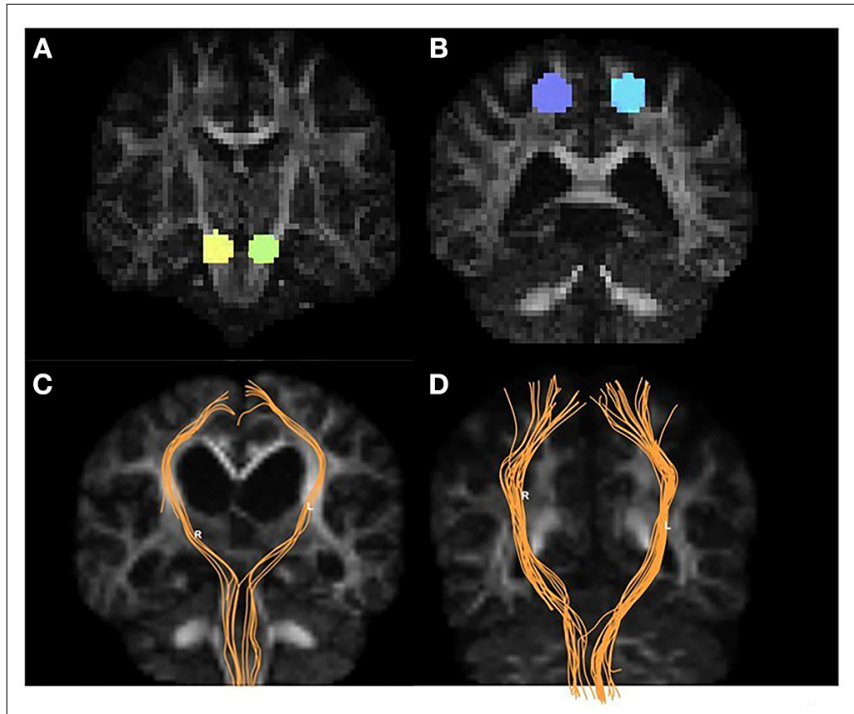

FIGURE 3 | Examples of volumes of interest, CST tracking, and CST reconstruction. Panel (A) shows the targets placed on two sides of the cerebral peduncle (yellow and green). Panel (B) shows the seeds placed on the precentral gyrus (purple and blue). The CST tractography (orange) for iNPH patients (C) and HCs (D).

parameters, the $\mathrm{MD}, \mathrm{AD}$, and $\mathrm{RD}$ values showed significant differences between iNPH patients and the HC group, while the FA values showed no significant differences between them.

Our study shows that QA values of bilateral corticospinal tracts were decreased in patients with iNPH compared with the QA values of HCs. Meanwhile, we found that there was no significant difference in the GFA values of corticospinal tracts in iNPH patients. GFA and QA values both represent the degree of anisotropy of the fiber bundles in DSI parameters. Some studies have suggested GFA as the main quantitative indicator 
TABLE 2 | Values measured in corticospinal tracts and P values of the iNPH patients and HCs.

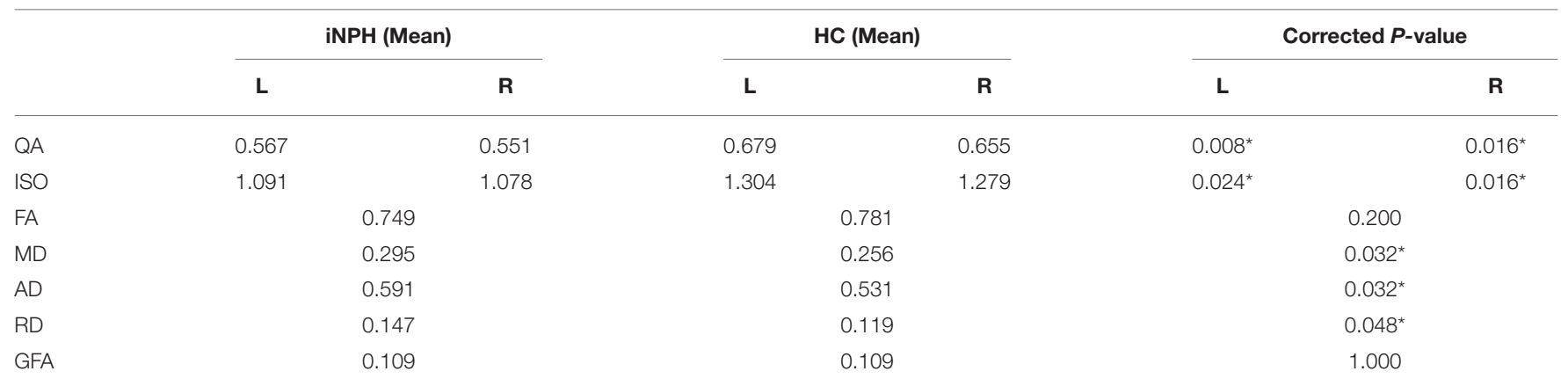

QA indicates quantitative anisotropy; ISO, isotropic diffusion component; $F A$, fractional anisotropy; $M D$, mean diffusivity; $A D$, axial diffusivity; RD, radial diffusivity; GFA, general fractional anisotropy. ${ }^{~} P<0.05$.
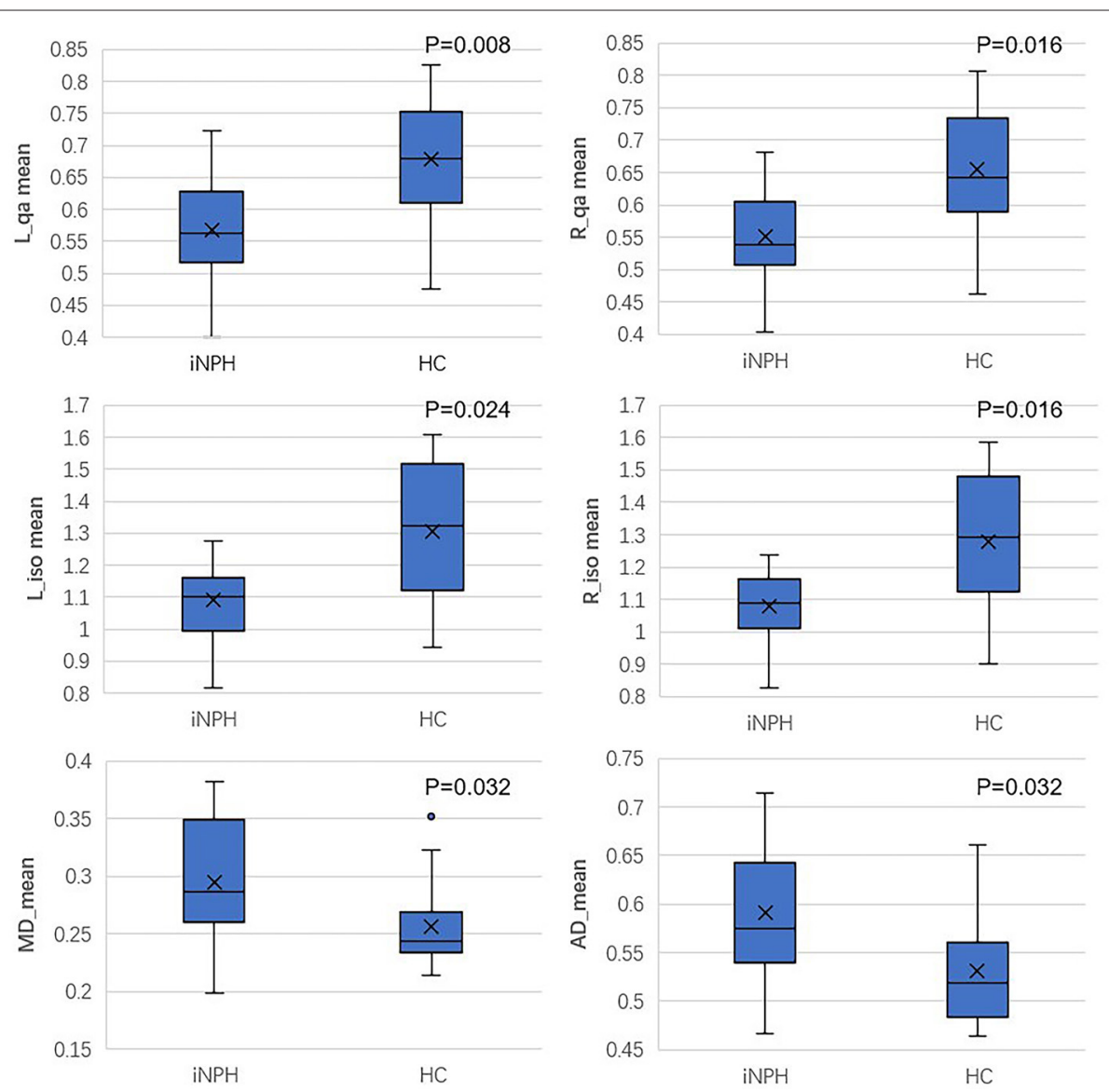

FIGURE 4 | Boxplot of QA, ISO, MD, and AD values between iNPH patients and HCs.

of DSI to use for analyses (11). However, several studies have demonstrated that GFA is easily sensitive to the partial volume effects of crossing fibers and edema, suggesting that it is not a stable index $(22,23)$. In fact, iNPH patients usually have periventricular white matter hyperintensities and periventricular edema. Thus, we suggest that GFA might not be a good parameter for evaluating changes in CSTs in iNPH patients. In contrast, the QA value is calculated from the reconstruction model of generalized q-sampling imaging (GQI) using DSI (24), which separates the ISO components of the fibers. Thus, QA is not susceptible to edema and crossing fibers but is more suitable for the analysis of hydrocephalus because it is always influenced by exudation. This allows QA values to reflect the changes in the fiber itself more accurately and reliably. A reduction in QA values reflects the damage to the integrity of the axon or myelin sheath in principle (18). 
In addition, the ISO values represent the isotropic diffusion of water molecules within cells or between cells, which is specific in DSI analysis in principle. We found that the ISO of the patient group was significantly lower than that of the control group. Combining QA and ISO, we suggested that this was possibly because the intercellular space was smaller and the isotropic diffusion of water molecules was restricted, which was caused by the compression of the lateral ventricle enlargement.

Studies of directional diffusion parameters such as FA values were found to be elevated in the corticospinal tracts of patients with iNPH (8). The FA values also showed significant differences in the axons of white matter in iNPH patients compared with patients with neurodegenerative diseases and healthy groups in previous studies (25). Our study found that the MD and $\mathrm{AD}$ values increased in iNPH patients, which is consistent with the results in the literature that implied an increase in the diffusion of water molecules. The increased MD and AD values could be caused by periventricular edema in iNPH patients, in accordance with a previous study observing that periventricular edema can lead to increased diffusion (26). However, our results show that the FA values were not significantly different between patients with iNPH and HCs, which is different from the findings of previous studies. According to the previous perspective, the enlarged lateral ventricles mechanically compressed the axons of corticospinal tracts and made the water molecules align along the axon orientation, which caused the FA values to be elevated $(27,28)$. In fact, mechanical pressure may cause atrophy and irreversible damage of the axons (21), which make FA values decrease accordingly. Combined with the above DSI parameters and FA in principle, we hypothesized that the lack of difference in FA values in our study was due to the coexistence of compression and injury. Thus, the variation in FA values could be canceled out.

Gait disturbance is always the first and foremost symptom in iNPH patients and is considered to be related to the injury to corticospinal tracts (29). The whole corticospinal tract is composed of complex fibers and intersects with many other fibers. We compared the diffusion parameters in CSTs of iNPH patients with those of age-matched healthy individuals. Our results show that the QA was significantly decreased in the patient group, but the most commonly used DTI parameter FA presented no significant difference between the two groups. The DTI model is unable to address such complex areas with various fiber directions, so it has some limitations in the analysis of white matter (30). DSI counterbalances the deficiency of DTI and has the ability to reveal crossing fibers, which can be used to show the complex neural network connectivity and microstructure of the brain $(31,32)$. DSI indicators were able to reflect the microscopic changes in the fiber structure more accurately. Our studies also found a difference in QA in the two hemispheres of CSTs in normal people. We suggest that this difference might be influenced by the difference between the dominant hemispheres. Nevertheless, these discrepancies were not observed in DTI indicators. Although there are other diffusion imaging studies in iNPH, this is the first DSI study providing a new diffusion method and complementary parameters for investigating alterations in nerve fibers in iNPH patients, enabling to widen the DTI findings.

This study also has some limitations. The relatively small sample size from a single center might limit the intergroup differences of some parameters, thereby preventing statistical significance from being achieved to a certain extent. This is a preliminary study, and longitudinal research on patients with iNPH in the future will be helpful in the preoperative evaluation of patient outcomes and can verify the accuracy of this study. Future research endeavors should be expanded to include more elderly people with neurodegenerative diseases who have some clinical manifestations similar to iNPH.

\section{CONCLUSION}

We found that iNPH patients present with decreased quantitative anisotropy, decreased isotropic diffusion component and increased mean diffusivity, radial diffusivity, and axial diffusivity in their corticospinal tracts. This reflected not only the compression of fibers by enlarged ventricles but also the change in the integrity of microstructural fibers, which helped us to better understand the mechanism of the disease.

\section{DATA AVAILABILITY STATEMENT}

The raw data supporting the conclusions of this article will be made available by the authors, without undue reservation.

\section{ETHICS STATEMENT}

The studies involving human participants were reviewed and approved by Research Ethics Committee of the First Affiliated Hospital of Shenzhen University. The patients/participants provided their written informed consent to participate in this study.

\section{AUTHOR CONTRIBUTIONS}

HZ, W-JH, FL, and YL: guarantors of integrity of entire study and manuscript editing. $\mathrm{HZ}$ and $\mathrm{W}-\mathrm{JH}$ : study conception/design, data analysis/interpretation and manuscript drafting, or revision for important intellectual content. HZ, W-JH, H-WZ, and L-HL: literature research and data analysis. HZ, S-PL, X-JZ, and LZ: data acquisition or clinical studies. All authors: approval of final version of submitted manuscript and agrees to ensure any questions related to the work are appropriately resolved.

\section{FUNDING}

This study was supported by a grant from Basic Plan Program of Shenzhen, China (No. JCYJ20180228163333734). 


\section{REFERENCES}

1. Mori E, Ishikawa $M$, Kato $T$, Kazui $H$, Miyake $H$, Miyajima $M$, et al. Guidelines for management of idiopathic normal pressure hydrocephalus: second edition. Neurol Med Chir (Tokyo). (2012) 52:775-809. doi: $10.2176 / \mathrm{nmc} .52 .775$

2. Adams DR, Fisher CM, Hakim S, Ojemann RG, Sweet WH. Symptomatic occult hydrocephalus with "normal" cerebrospinal-fluid pressure. A treatable syndrome. N Engl J Med. (1965) 273:117-26. doi: 10.1056/NEJM196507152730301

3. Hakim S, Adams RD. The special clinical problem of symptomatic hydrocephalus with normal cerebrospinal fluid pressure. Observations on cerebrospinal fluid hydrodynamics. J Neurol Sci. (1965) 2:307-27. doi: 10.1016/0022-510X(65)90016-X

4. Toma AK, Holl E, Kitchen ND, Watkins LD. Evans' index revisited: the need for an alternative in normal pressure hydrocephalus. Neurosurgery. (2011) 68:939-44. doi: 10.1227/NEU.0b013e318208f5e0

5. Quattrone A, Sarica A, La Torre D, Morelli M, Vescio B, Nigro S, et al. Magnetic resonance imaging biomarkers distinguish normal pressure hydrocephalus from progressive supranuclear palsy. Mov Disord. (2020) 35:1406-15. doi: 10.1002/mds.28087

6. Williams MA, Relkin NR. Diagnosis and management of idiopathic normal-pressure hydrocephalus. Neurol Clin Pract. (2013) 3:375-85. doi: 10.1212/CPJ.0b013e3182a78f6b

7. Bradley WG. Normal pressure hydrocephalus: new concepts on etiology and diagnosis. AJNR Am J Neuroradiol. (2000) 21:1586-90.

8. Jurcoane A, Keil F, Szelenyi A, Pfeilschifter W, Singer OC, Hattingen E. Directional diffusion of corticospinal tract supports therapy decisions in idiopathic normal-pressure hydrocephalus. Neuroradiology. (2014) 56:5-13. doi: 10.1007/s00234-013-1289-8

9. Wiegell MR, Larsson HB, Wedeen VJ. Fiber crossing in human brain depicted with diffusion tensor MR imaging. Radiology. (2000) 217:897-903. doi: 10.1148/radiology.217.3.r00nv43897

10. Schmahmann JD, Pandya DN, Wang R, Dai G, D'Arceuil HE, de Crespigny AJ, et al. Association fibre pathways of the brain: parallel observations from diffusion spectrum imaging and autoradiography. Brain. (2007) 130 (Pt 3):630-53. doi: 10.1093/brain/awl359

11. Lin YC, Shih YC, Tseng WY, Chu YH, Wu MT, Chen TF, et al. Cingulum correlates of cognitive functions in patients with mild cognitive impairment and early Alzheimer's disease: a diffusion spectrum imaging study. Brain Topogr. (2014) 27:393-402. doi: 10.1007/s10548-013-0346-2

12. Wu CH, Hwang TJ, Chen PJ, Chou TL, Hsu YC, Liu CM, et al. Reduced structural integrity and functional lateralization of the dorsal language pathway correlate with hallucinations in schizophrenia: a combined diffusion spectrum imaging and functional magnetic resonance imaging study. Psychiatry Res. (2014) 224:303-10. doi: 10.1016/j.pscychresns.2014.08.010

13. Romascano D, Meskaldji DE, Bonnier G, Simioni S, Rotzinger D, Lin YC, et al. Multicontrast connectometry: a new tool to assess cerebellum alterations in early relapsing-remitting multiple sclerosis. Hum Brain Mapp. (2015) 36:1609-19. doi: 10.1002/hbm.22698

14. Lin HY, Perry A, Cocchi L, Roberts JA, Tseng WI, Breakspear M, et al. Development of frontoparietal connectivity predicts longitudinal symptom changes in young people with autism spectrum disorder. Transl Psychiatry. (2019) 9:86. doi: 10.1038/s41398-019-0418-5

15. Wedeen VJ, Wang RP, Schmahmann JD, Benner T, Tseng WY, Dai $\mathrm{G}$, et al. Diffusion spectrum magnetic resonance imaging (DSI) tractography of crossing fibers. Neuroimage. (2008) 41:1267-77. doi: 10.1016/j.neuroimage.2008.03.036

16. Leng B, Han S, Bao Y, Zhang H, Wang Y, Wu Y, et al. The uncinate fasciculus as observed using diffusion spectrum imaging in the human brain. Neuroradiology. (2016) 58:595-606. doi: 10.1007/s00234-016-1650-9

17. Basser PJ. Inferring microstructural features and the physiological state of tissues from diffusion-weighted images. NMR Biomed. (1995) 8:333-44. doi: 10.1002/nbm.1940080707

18. Yeh FC, Wedeen VJ, Tseng WY. Generalized q-sampling imaging. IEEE Trans Med Imaging. (2010) 29:1626-35. doi: 10.1109/TMI.2010.2045126
19. Shinoda N, Hirai O, Hori S, Mikami K, Bando T, Shimo D, et al. Utility of MRI-based disproportionately enlarged subarachnoid space hydrocephalus scoring for predicting prognosis after surgery for idiopathic normal pressure hydrocephalus: clinical research. J Neurosurg. (2017) 127:1436-42. doi: 10.3171/2016.9.JNS161080

20. Yeh FC, Liu L, Hitchens TK, Wu YL. Mapping immune cell infiltration using restricted diffusion MRI. Magn Reson Med. (2017) 77:603-12. doi: $10.1002 / \mathrm{mrm} .26143$

21. Ades-Aron B, Yeager S, Miskin N, Fieremans E, George A, Golomb J. Diffusional kurtosis along the corticospinal tract in adult normal pressure hydrocephalus. AJNR Am J Neuroradiol. (2018) 39:2218-23. doi: 10.3174/ajnr.A5845

22. Yeh FC, Verstynen TD, Wang Y, Fernández-Miranda JC, Tseng WY. Deterministic diffusion fiber tracking improved by quantitative anisotropy. PLoS One. (2013) 8:e80713. doi: 10.1371/journal.pone.008 0713

23. Faraji AH, Abhinav K, Jarbo K, Yeh FC, Shin SS, Pathak S, et al. Longitudinal evaluation of corticospinal tract in patients with resected brainstem cavernous malformations using high-definition fiber tractography and diffusion connectometry analysis: preliminary experience. J Neurosurg. (2015) 123:1133-44. doi: 10.3171/2014.12.JNS142169

24. Menzel MI, Tan ET, Khare K, Sperl JI, King KF, Tao X, et al. Accelerated diffusion spectrum imaging in the human brain using compressed sensing. Magn Reson Med. (2011) 66:1226-33. doi: 10.1002/mrm.23064

25. Kang K, Choi W, Yoon U, Lee JM, Lee HW.Abnormal white matter integrity in elderly patients with idiopathic normal-pressure hydrocephalus: a tractbased spatial statistics study. Eur Neurol. (2016) 75:96-103. doi: 10.1159/000 443206

26. Ebisu T, Naruse S, Horikawa Y, Ueda S, Tanaka C, Uto M, et al. Discrimination between different types of white matter edema with diffusion-weighted MR imaging. J Magn Reson Imaging. (1993) 3:863-8. doi: 10.1002/jmri.1880030612

27. Assaf Y, Ben-Sira L, Constantini S, Chang LC, Beni-Adani L. Diffusion tensor imaging in hydrocephalus: initial experience. AJNR Am J Neuroradiol. (2006) 27:1717-24.

28. Hattingen E, Jurcoane A, Melber J, Blasel S, Zanella FE, NeumannHaefelin $\mathrm{T}$, et al. Diffusion tensor imaging in patients with adult chronic idiopathic hydrocephalus. Neurosurgery. (2010) 66:917-24. doi: 10.1227/01.NEU.0000367801.35654.EC

29. Bradley WG Jr, Whittemore AR, Kortman KE, Watanabe AS, Homyak M, Teresi LM, et al. Marked cerebrospinal fluid void: indicator of successful shunt in patients with suspected normal-pressure hydrocephalus. Radiology. (1991) 178:459-66. doi: 10.1148/radiology.178.2.1987609

30. Oouchi H, Yamada K, Sakai K, Kizu O, Kubota T, Ito H, et al. Diffusion anisotropy measurement of brain white matter is affected by voxel size: underestimation occurs in areas with crossing fibers. AJNR Am J Neuroradiol. (2007) 28:1102-6. doi: 10.3174/ajnr.A0488

31. Wedeen VJ, Hagmann P, Tseng WY, Reese TG, Weisskoff RM. Mapping complex tissue architecture with diffusion spectrum magnetic resonance imaging. Magn Reson Med. (2005) 54:1377-86. doi: 10.1002/mrm. 20642

32. Wedeen VJ, Rosene DL, Wang R, Dai G, Mortazavi F, Hagmann P, et al. The geometric structure of the brain fiber pathways. Science. (2012) 335:1628-34. doi: $10.1126 /$ science. 1215280

Conflict of Interest: The authors declare that the research was conducted in the absence of any commercial or financial relationships that could be construed as a potential conflict of interest.

Copyright (C) 2021 Zhang, He, Liang, Zhang, Zhang, Zeng, Luo, Lin and Lei. This is an open-access article distributed under the terms of the Creative Commons Attribution License (CC BY). The use, distribution or reproduction in other forums is permitted, provided the original author(s) and the copyright owner(s) are credited and that the original publication in this journal is cited, in accordance with accepted academic practice. No use, distribution or reproduction is permitted which does not comply with these terms. 